\section{SP0091 US FOR PERIPHERAL NERVE ENTRAPMENTS + DEMO}

D.A. Bong ${ }^{1,2}$, on behalf of Eular Working Group-anatomy for the Image. ${ }^{1}$ Instituto Poal de Reumatologia, Barcelona; ${ }^{2}$ Facultat de Medicina, Universitat de Barcelona, Spain

Symptoms related to entrapment of the peripheral nerves of the upper and lower extremity are common in rheumatologic practice and are often misinterpreted as articular or non neurologic periarticular pathology. Owing to the varible trajectory and mobility of the peripheral nerves, high resolution musculoskeletal ultrasound (US) has many advantages over other imaging modalities including superior resolution, ability to perform a dynamic real-time examination, precise sonopalpation and comparison with the contralateral structures. Its availability, relative ease of application in experienced hands and tolerance argue for its use as the initial "intervention" when compared to electrophysiologic testing. Large, medium and many smaller nerves can be imaged directly (along with the musculoskeletal terrain through which they pass ) depending on the quality of the instrumentation, skill and anatomic knowledge of the sonographer. Even pathologic involvement of smallest "nonvisualized" small nerves may be deduced by secondary changes of the innervated musculature, the so-called "echo-myogram". Obviously a thorough knowledge of the peripheral neuroanatomy and musculuskeletal relationships along the nerve trajectory is the key to an enhanced and reliable evaluation. This presentation will focus on the principles of neuro-sonoanatomy of the major peripheral nerve of the extremities and the fundamentals of the peripheral nerve US examination will be demonstrated.

Disclosure of Interest: None declared

DOI: 10.1136/annrheumdis-2018-eular.7856

\section{SP0092 US FOR SCORING SJÖGREN DISEASE}

S. Jousse-Joulin, on behalf of omeract task force. rheumatology, cavale blanche hospital, brest, France

Sjogren's syndrome (SS) is a chronic autoimmune inflammatory disorder of exocrine glands. Its diagnosis relies on solely a combination of clinical and laboratory findings. Recently, new imaging techniques as MRI or ultrasound, known to be less invasive than sialography have shown a good sensitivity and specificity to detect structural abnormalities in pSS. Ultrasonography of the salivary glands (SGUS) appears to be the most attractive imaging approach: it is an inexpensive commonly available noninvasive technique that does not cause complications and inconvenience to the patient, although the data are somewhat conflicting. A systematic literature review has highlighted the main keypoints to validate salivary glands ultrasonography has an outcome measure in particular, there are limited data describing a standardised scanning method and standardised definitions of US gland pathologies. 'Until now, both parotid glands and submandibular glands were assessed using a scoring system first developed by de Vita et al.( , $^{2}$ later modified by other investigators. Five scoring systems are now published which are frequently modified by different operators ${ }^{2,6}$ but their reliability were not always evaluated. In order to use SGUS in clinical routine, we need to have standardisation of the US procedure to assess SG and to be capable to have a simple and reliable score to rate structural damage of each. A SGUS OMERACT task force was created in 2016 and produce consensual US definitions of the 6 major salivary glands and a semi quantitative scoring. The intra and inter reliability of this scoring between international experts showed good inter reliability and excellent intra reliability. We will present to you the definitions and the new semi quantitative scoring with a live demo.

\section{REFERENCES:}

[1] Jousse-Joulin S, et al. Rheumatology (Oxford) 2016 May;55(5):789-800.

[2] De vita S, et al. J rheumatol 2000 May;27(5):1229-36.

[3] Milic V, et al. Rheumatol 2012 Jun;51(6):1081-5.

[4] Makula E, et al. Br J Rheumatol1996 Oct;35(10):972-7.

[5] Sallaffi F, et al. J Rheumatol 2000 May;27(5):1229-36.

[6] Hocevar A, et al. Eur J radiol 2007 Sep;63(3):379-83.

Disclosure of Interest: None declared DOI: 10.1136/annrheumdis-2018-eular.7826
THURSDAY, 14 JUNE 2018

\section{Immune senescence and ageing}

\section{SP0093 RESETTING THE IMMUNE SYSTEM IN AUTOIMMUNITY}

F. Van Wijk. Laboratory of Translational Immunology, University Medical Centre Utrecht, Utrecht, Netherlands

Autologous hematopoietic stem cell transplantation (HSCT) is a last resort treatment for refractory autoimmune patients and the only therapy so far that can induce long-term drug-free remission in these patients. Understanding the mechanisms responsible for this regained immune tolerance may help to develop other, less aggressive immune-mediated interventions with a similar outcome. Although the underlying mechanisms are incompletely understood, extensive immune ablation followed by autologous stem cell infusion seems to be able to rewire a faulty immune system. It is so far unknown which cells need to be removed prior to autologous transplantation and which cells are important in controlling disease after transplantation. We have previously shown that transplantation restores immune tolerance by renewal and modulation of both the CD4 effector T cell (Teff) and FOXP3 Treg compartment in a proteoglycan induced arthritis (PGIA) mouse model. In the human setting we further looked into T cell renewal by TCR CDR3 b chain repertoire sequencing prior to and post HSCT of juvenile idiopathic arthritis and dermatomyositis patients. We found that TCR beta chain diversity of Treg was highly restricted prior to transplantation and that the TCR b chain diversity of Treg increased post-transplantation. The TCR beta chain diversity of the CD4 non-Treg compartment also expanded after aSCT, although not as strikingly as the Treg compartment, indicating that the Treg compartment is more affected. Interestingly, in patients these highly dominant T(reg) cell clones persist over time and (locally) expand with every relapse of disease. The question is now how HSCT or related therapies can efficiently target and stimulate $\mathrm{T}$ cell renewal in chronic autoimmune inflammation, with limited toxicity.

Disclosure of Interest: None declared

DOI: 10.1136/annrheumdis-2018-eular.7703

FRIDAY, 15 JUNE 2018

Special delivery: Intercellular communication

\section{SP0094 NEW INSIGHTS INTO ANTIBODY-BASED THERAPIES AND DISEASE SUSCEPTIBILITIES REVEALED BY MICROSCOPY OF HUMAN NK CELLS}

D.M. Davis. Manchester Collaborative Centre for Inflammation Research, University of Manchester, Manchester, UK

Background: Natural Killer (NK) cells express the Fc receptor CD16 (FcyRIIla), which can trigger antibody-dependant cellular cytotoxicity (ADCC) against opsonized cells. ADCC is clinically important as one of the mechanisms by which therapeutic antibodies work. The anti-CD20 mAb rituximab is, for example, widely used for targeting B cells in treatments of autoimmune disease and non-Hodgkin's lymphoma. Here, we set out to study individual NK cell-target cell interactions by microscopy to determine what happens as NK cells meet different target cells sequentially, some opsonised and some not.

In addition, genetic diversity in human NK cell receptors has been linked with resistance and susceptibility to many diseases, but underlying mechanisms remain unclear. The effect of this genetic diversity on the cell surface organisation and signalling of receptors is a major unknown. Thus, we set out to study the organisation and signalling of inhibitory Killer lg-like receptors (KIR) encoded by different genes and alleles using super-resolution microscopy.

Results: We found that repeated activation via CD16 decreased the amount of perforin secreted. However, perforin secretion was restored upon subsequent activation via a different NK cell activating receptor, NKG2D. Repeated stimulation via NKG2D also decreased perforin secretion but this was not rescued by stimulation via CD16. These different outcomes of sequential stimulation could be accounted for by shedding of CD16 being triggered by cellular activation. Shedding of CD16 shedding also increased NK cell motility and allowed for detachment of NK cells from target cells. In turn, this aided NK cell survival and boosted serial engagement of target cells.

In addition, we report that inhibitory NK cell receptors encoded by different genes and alleles organise differently at the surface of primary human NK cells. In particular, expression level has major effects on receptor organisation. KIR that are expressed at a low level assemble in smaller clusters than KIR that are highly expressed. Unexpectedly, upon receptor triggering, low-expressed receptors in smaller clusters generate more phosphorylated Crk than highly expressed receptors. 